\title{
Synthesis of fluorescent dibenzopyranones by the Diels-Alder reaction of 4-styrylcoumarins and $N$-phenylmaleimide and in situ aromatization using DDQ
}

\author{
Kailas K. Sanap and Shriniwas D. Samant* \\ Department of Chemistry, Institute of Chemical Technology, Matunga, Mumbai-400 019 India \\ E-mail: samantsd@yahoo.com, samantsd.ict@gmail.com
}

\begin{abstract}
Reaction of 7-substituted-4-styrylcoumarins and $\mathrm{N}$-phenylmaleimide in nitrobenzene under reflux conditions affords mainly 7-substituted-2,11-diphenyl-3a,10,11,11a-tetrahydro[1]benzopyrano[3,4-e]isoindole-1,3,4(2H)-triones. The same reaction in $o$-dichlorobenzene in the presence of DDQ gives the corresponding aromatized dibenzopyranones. The dibenzopyranones are fluorescent and their UV and fluorescence spectra are reported.
\end{abstract}

Keywords: Diels-Alder reaction, 4-styrylcoumarin, dibenzopyranone

\section{Introduction}

The dibenzopyranone unit is present in many natural products, ${ }^{1-5}$ such as alternariol, graphislactones, autumnariol, autumnariniol, and altenuisol, and in many biologically active compounds. ${ }^{6-7}$ Such lactones have been used as intermediates in the synthesis of several pharmaceutically interesting compounds including progesterone, androgen, glucocorticoid modulators, ${ }^{8-10}$ and endothelial cell proliferation inhibitors. ${ }^{11}$ Furthermore, dibenzopyranones occur naturally in many food sources including citrus fruits, herbs, and vegetables. ${ }^{12}$ There are several methods available for the synthesis of dibenzopyranones, the most common being Suzuki cross-coupling reaction followed by Lewis $\operatorname{acid}^{13-16}$ or metal ${ }^{17}$ mediated lactonization. More recently, the Diels-Alder cycloaddition of 4-cyanocoumarins (as dienophiles), ${ }^{18}$ inverse electron demand Diels-Alder reaction of coumarin fused electron deficient dienes with electron rich dienophiles, ${ }^{19-21}$ tert-butyllithium-mediated cyclization of bromobenzylfluorophenyl ethers, ${ }^{22}$ and ruthenium-catalyzed cyclotrimerization of aryl diynes ${ }^{23}$ have been reported. In 2002, Abbott Laboratories reported a practicable and scalable synthesis of glucocorticoid receptor A-224817.0 through Negishi cross coupling. ${ }^{24}$ However, the disadvantages of these methods include low overall yield, long reaction time, low temperature conditions, multistep sequence, and the need to purify the intermediates. 
Diels-Alder reaction of 4-styrylcoumarins with $N$-phenylmaleimide in nitrobenzene is reported to give 7-substituted-2,11-diphenyl-3a,10,11,11a-tetrahydro[1]benzopyrano[3,4-e]isoindole-1,3,4(2H)-triones, and no further dehydrogenation is observed. ${ }^{25-27}$ Diels-Alder reaction of 4-styrylcoumarins gives an entry to dibenzopyranones. We are hereby reporting a simple route for the synthesis of dibenzopyranones via a tandem Diels-Alder reactiondehydrogenation of 4-styrylcoumarins (1) and $N$-phenylmaleimide (NPMA) (2) in the presence of DDQ.

\section{Results and Discussion}

7-Methyl-4-styrylcoumarin (1a) was prepared by a known method. ${ }^{28} \mathbf{1 a}$ was subjected to DielsAlder reaction with $N$-phenylmaleimide (2) (NPMA) in boiling nitrobenzene when adduct 3a was formed within $10 \mathrm{~min}$. When the reaction was continued further, a small amount of the corresponding dehydrogenated aromatic product $\mathbf{4 a}$ was formed along with 3a. The reaction was continued up to $24 \mathrm{~h}$ to see whether compound 3a underwent retro Diels-Alder reaction. Instead, 3a underwent dehydrogenation slowly to form $\mathbf{4 a}$ indicating a nitrobenzene promoted dehydrogenation of 3a. This was confirmed by aniline produced in the reaction. Dehydrogenation of hydroaromatics under neutral conditions requires high temperature and a catalyst. ${ }^{29-33}$ Nitrobenzene is a known dehydrogenating agent, e.g. as in the Skraup synthesis. ${ }^{34}$ In the ${ }^{1} \mathrm{H}$ NMR, 4a showed a multiplet at 7.29-7.55 $\delta$ corresponding to aromatic $12 \mathrm{H}$, a doublet at $8.02 \delta$ for $\mathrm{C}_{1} \underline{\mathrm{H}}$, and a singlet at $8.35 \delta$ for $\mathrm{C}_{10} \underline{\mathrm{H}}$, indicating the dehydrogenation of 3a to 4a. No signal was observed for aliphatic protons.

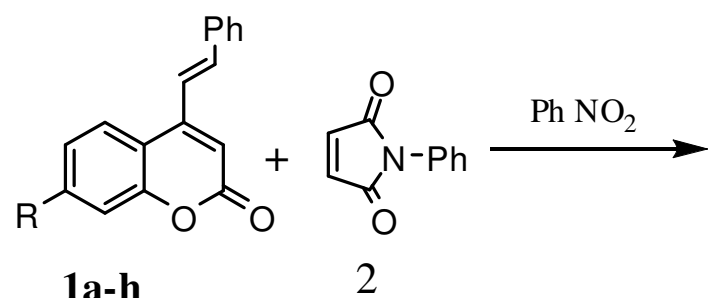

1a: $\mathrm{R}=\mathrm{Me}$

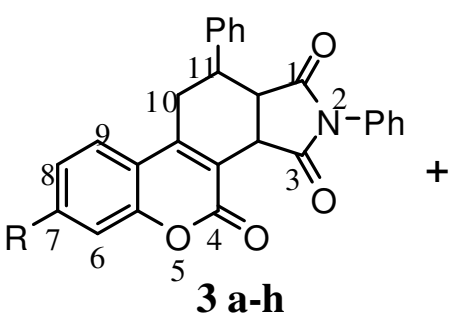

3a: $\mathrm{R}=\mathrm{Me}$

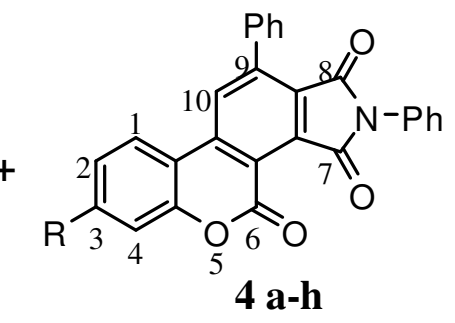

4a: $\mathrm{R}=\mathrm{Me}$

Scheme 1. Diels-Alder reaction of 4-styrylcoumarins with $N$-phenylmaleimide in nitrobenzene.

The dehydrogenation step, i.e. 3 to $\mathbf{4}$, was studied further in order to confirm the role of nitrobenzene. 3a was heated separately in boiling nitrobenzene. The dehydrogenation reaction was very slow and only $9 \%$ of $\mathbf{4 a}$ was formed in $24 \mathrm{~h}$. The Diels-Alder adducts $\mathbf{3 b}(\mathrm{R}=\mathrm{OH}), \mathbf{3 c}$ $(\mathrm{R}=\mathrm{OMe})$ and $\mathbf{3 d}(\mathrm{R}=\mathrm{H})$ also gave the corresponding dehydrogenated products $\mathbf{4 b}, \mathbf{4 c}$ and $\mathbf{4 d}$ respectively, in low yield on heating in boiling nitrobenzene (Table 1). 
Table 1. Dehydrogenation of Diels-Alder adducts (3) in nitrobenzene ${ }^{\mathrm{a}}$

\begin{tabular}{ccccc}
\hline Entry & Compound & Time $^{\mathrm{b}}(\mathrm{h})$ & Product & Yield $^{\mathrm{c}}(\%)$ \\
\hline 1 & $\mathbf{3 a}$ & 24 & $\mathbf{4 a}$ & 9 \\
2 & $\mathbf{3 b}$ & 36 & $\mathbf{4 b}$ & 13 \\
3 & $\mathbf{3 c}$ & 36 & $\mathbf{4 c}$ & 14 \\
4 & $\mathbf{3 d}$ & 36 & $\mathbf{4 d}$ & 14 \\
\hline
\end{tabular}

Reaction conditions: $\mathbf{3}(1 \mathrm{mmol})$; nitrobenzene $(5 \mathrm{~mL})$; ${ }^{\mathrm{a}}$ Reflux temperature;

${ }^{\mathrm{b}}$ Time for which reaction was continued; ${ }^{\mathrm{c}}$ Isolated yield.

In order to see the effect of NPMA on the dehydrogenation, we carried out the Diels-Alder reaction with an increasing amount of NPMA. However, the quantity of NPMA was not found to have any effect on the dehydrogenation (Table 2).

Table 2. Effect of increasing quantity of NPMA in the reaction with 1a on the yield of 4a

\begin{tabular}{cccc}
\hline Entry & Equivalent of $\mathbf{2}$ & Time $^{\mathrm{b}}(\mathrm{h})$ & Yield $^{\mathrm{c}}$ of $\mathbf{4 a}(\%)$ \\
\hline 1 & 2 & 24 & 6 \\
2 & 3 & 24 & 9 \\
3 & 4 & 24 & 9 \\
4 & 5 & 24 & 10 \\
5 & 6 & 24 & 10 \\
\hline
\end{tabular}

Reaction conditions: 1a $(0.262 \mathrm{~g}, 1 \mathrm{mmol})$; nitrobenzene: $5 \mathrm{~mL}$; Temp: Reflux; ${ }^{\mathrm{b}}$ Time for which reaction was continued; ${ }^{\mathrm{C}}$ Isolated yield.

The dehydrogenation of 3a was attempted in the presence of $\mathrm{Pd} / \mathrm{C}(10 \%)$, sulfur, napthoquinone, and DDQ, in different solvents (Table 3). DDQ in nitrobenzene and $o$ dichlorobenzene (o-DCB) was found to be the most effective dehydrogenating agent. In xylene, 3a was partly soluble at the reflux temperature and only a trace of the product was obtained.

Our next objective was to develop a one-pot process, where the Diels-Alder reaction and dehydrogenation would occur subsequently. 1a was reacted with NPMA in boiling nitrobenzene or o-dichlorobenzene, in the presence of DDQ by varying the mole ratio for NPMA and DDQ (Table 4). Two equivalents of DDQ and three equivalents of NPMA in o-DCB gave $73 \%$ yield of $\mathbf{4 a}$ in $1 \mathrm{~h}$. In nitrobenzene, the required time for Diels-Alder-dehydrogenation reaction was shorter, but the yield obtained was $48 \%$ and $49 \%$ using two and three equivalents of DDQ, respectively. Surprisingly, though the Diels-Alder reaction in o-DCB was slower as compared to nitrobenzene, the yield in o-DCB was better. 
Table 3. Conversion of $\mathbf{3 a}$ to $\mathbf{4 a}$ using of different dehydrogenating reagents and solvents

\begin{tabular}{|c|c|c|c|c|}
\hline Entry & $\begin{array}{c}\text { Reagent } \\
\text { (equiv.) }\end{array}$ & Solvent $^{\mathrm{a}}$ & $\begin{array}{c}\text { Time }^{b} \\
(h)\end{array}$ & $\begin{array}{c}\text { Yield }^{\mathrm{c}} \text { of } \\
\mathbf{4 a}(\%)\end{array}$ \\
\hline 1 & $\mathrm{Pd} / \mathrm{C}(10 \%),(20 \mathrm{wt} \%)$ & Glacial AcOH & 12 & 21 \\
\hline 2 & $\mathrm{Pd} / \mathrm{C}(10 \%),(30 \mathrm{wt} \%)$ & Glacial AcOH & 18 & 27 \\
\hline 3 & Sulfur (4) & Nitrobenzene & 18 & 36 \\
\hline 4 & Sulfur (4) & $o$-Dichlorobenzene & 18 & 29 \\
\hline 5 & Naphthoquinone (2) & Nitrobenzene & 18 & 27 \\
\hline 6 & DDQ (2) & Nitrobenzene & $5 \mathrm{~min}$ & 61 \\
\hline 7 & DDQ (2) & $o$-Dichlorobenzene & $5 \mathrm{~min}$ & 79 \\
\hline 8 & DDQ (2) & Xylene & 18 & Traces \\
\hline
\end{tabular}

Reaction conditions: 3a (0.435 g, $1 \mathrm{mmol})$; Solvent (5 mL); ${ }^{\mathrm{a}}$ Reflux temp; ${ }^{\mathrm{b}}$ Time for which reaction was continued; ${ }^{\mathrm{c}}$ Isolated yield.

Table 4. Diels-Alder reaction of 1a and NPMA using different mole ratios of NPMA and DDQ in nitrobenzene and o-dichlorobenzene (o-DCB)

\begin{tabular}{cccccc}
\hline Entry & $($ DDQ/NPMA $)$ & $\begin{array}{c}\text { Mole ratio } \\
\text { 1a: DDQ/NPMA }\end{array}$ & Solvent $^{\mathrm{a}}$ & $\begin{array}{c}\text { Time }^{\mathrm{b}} \\
(\mathrm{h})\end{array}$ & Yield $^{\mathrm{c}}$ of 4a $(\%)$ \\
\hline 1 & 1 & Nitrobenzene & 2 & 27 \\
2 & DDQ & 2 & Nitrobenzene & 15 min & 48 \\
3 & DDQ & 3 & Nitrobenzene & 15 min & 49 \\
4 & DDQ & 1 & $o$-DCB & 3 & 42 \\
5 & DDQ & 2 & $o$-DCB & 1 & 73 \\
6 & DDQ & 3 & $o$-DCB & 1 & 75 \\
7 & NPMA & 2 & $o$-DCB & 1.5 & 64 \\
8 & NPMA & 4 & $o$-DCB & 1 & 73 \\
9 & NPMA & 5 & $o$-DCB & 1 & 75 \\
\hline
\end{tabular}

Reaction conditions: 1a $(0.262 \mathrm{~g}, 1 \mathrm{mmol})$; Solvent $(5 \mathrm{~mL}) ;{ }^{\mathrm{a}}$ Reflux temperature; ${ }^{\mathrm{b}}$ Time for which reaction was continued; For entries (1-6) - NPMA used 3 equiv.; For entries (7-9) - DDQ used 2 equiv.; ${ }^{\mathrm{C}}$ Isolated yield.

Using the optimized conditions we carried out the reactions for other substrates to study the generality of tandem Diels-Alder-Dehydrogenation reactions. Different 4-styrylcoumarins were 
prepared by the known procedure. ${ }^{28}$ They were reacted with NPMA under the optimized conditions to obtain a series of dibenzopyranones (4) (Table 5).

Table 5. Tandem Diels-Alder-dehydrogenation reaction of $\mathbf{1}$ with NPMA in $o$-dichlorobenzene ${ }^{\mathrm{a}}$

\begin{tabular}{ccccc}
\hline Entry & Diene $\mathbf{1}$ & $(\mathrm{R})$ & Time $^{\mathrm{b}}(\mathrm{min})$ & Yield $^{\mathrm{c}}$ of $\mathbf{4}$ \\
\hline 1 & $\mathbf{1 a}$ & $\mathrm{Me}$ & 60 & 73 \\
2 & $\mathbf{1 b}$ & $\mathrm{OH}$ & 75 & 71 \\
3 & $\mathbf{1 c}$ & $\mathrm{OMe}$ & 75 & 69 \\
4 & $\mathbf{1 d}$ & $\mathrm{H}$ & 70 & 66 \\
5 & $\mathbf{1 e}$ & $\mathrm{OTs}$ & 85 & 61 \\
6 & $\mathbf{1 f}$ & $\mathrm{OAc}$ & 85 & 63 \\
7 & $\mathbf{1 g}$ & $\mathrm{Cl}$ & 80 & 67 \\
8 & $\mathbf{1 h}$ & $\mathrm{NHCO}_{2} \mathrm{Et}$ & 75 & 72 \\
\hline
\end{tabular}

Reaction conditions: mole ratio (1: NPMA) 1:3 equiv.; 1: DDQ 2 equiv.; ${ }^{\mathrm{a} R e f l u x}$ temperature; ${ }^{\mathrm{b}}$ Time for which reaction was continued; ${ }^{\mathrm{C}}$ Isolated yield.

Compounds 4 showed fluorescence. Hence, their UV absorption and emission properties were studied in chloroform + methanol (1:1, v/v) (Figure 1, Table 6). In the same solvent system standards anthracene and fluorescein show fluorescence at 488 and $513 \mathrm{~nm}$ respectively. UV ( $\left.\lambda_{\text {abs }} \max \right)$ and emission $\left(\lambda_{\mathrm{f}} \max \right)$ depended upon the nature of the substituent on the coumarin ring. High stoke shift ( $\Delta_{\lambda \mathrm{f}}$-abs) was observed in the case of $\mathbf{4 a}, \mathbf{4 b}$, and $\mathbf{4 c}$. This may be ascribed to the increased $\pi$-electron density on the coumarin ring, due to the presence of electron donating groups $-\mathrm{Me},-\mathrm{OH},-\mathrm{OCH}_{3}$. The other substituents showed poor stokes shift.
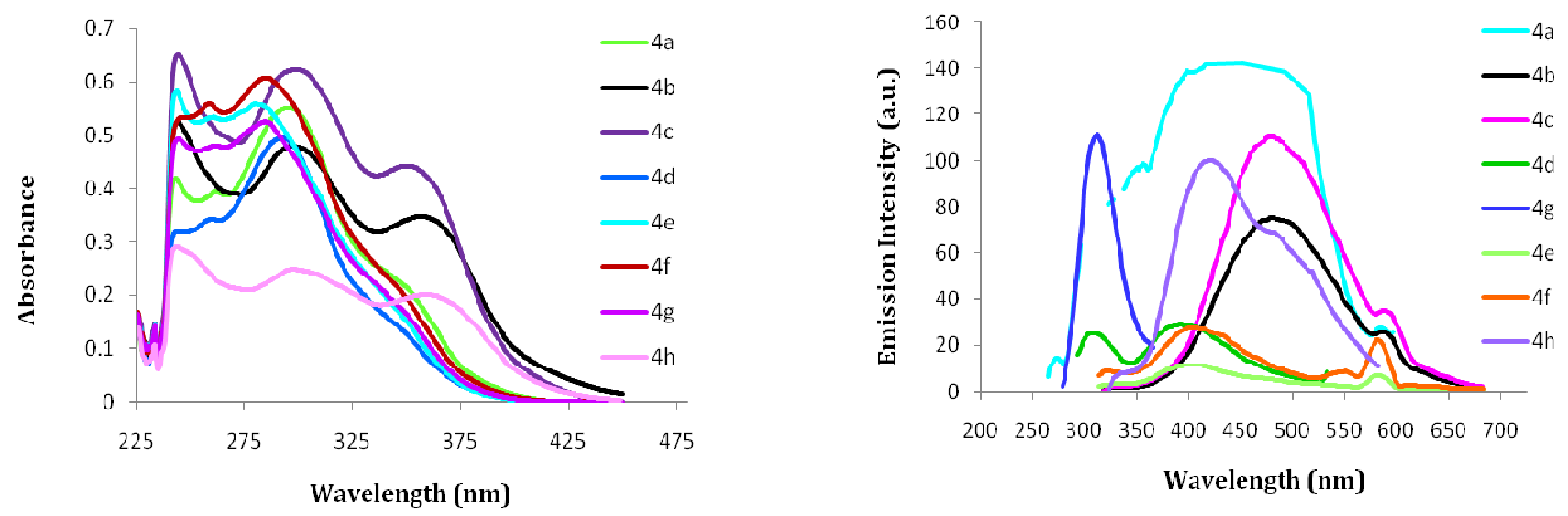

Figure 1. Absorption and emission spectra of $\mathbf{4 a - 4 h}$. 
Table 6. UV-Vis absorption $\left(\lambda_{\mathrm{abs}} \max \right)$ and fluorescence $\left(\lambda_{\mathrm{f}} \max \right)$ of 4

\begin{tabular}{ccccccc}
\hline Entry & Product & $\mathrm{R}$ & $\begin{array}{c}\lambda_{\text {abs }} \text { max } \\
(\mathrm{nm})\end{array}$ & $\begin{array}{c}\lambda_{\mathrm{f}} \max \\
(\mathrm{nm})\end{array}$ & $\begin{array}{c}\text { Extinction } \\
\text { coefficient } \\
\text { Lit mol }^{-1} \mathrm{~cm}^{-1}\end{array}$ & $\Delta \lambda \mathrm{f}_{\text {-abs }}(\mathrm{nm})$ \\
\hline 1 & $\mathbf{4 a}$ & $\mathrm{Me}$ & 295 & 452 & 23762 & 157 \\
2 & $\mathbf{4 b}$ & $\mathrm{OH}$ & 296 & 478 & 20745 & 182 \\
3 & $\mathbf{4 c}$ & $\mathrm{OMe}$ & 297 & 480 & 27805 & 183 \\
4 & $\mathbf{4 d}$ & $\mathrm{H}$ & 292 & 400 & 20634 & 108 \\
5 & $\mathbf{4 e}$ & $\mathrm{OTs}$ & 292 & 394 & 32826 & 102 \\
6 & $\mathbf{4 f}$ & $\mathrm{OAc}$ & 291 & 396 & 28862 & 105 \\
7 & $\mathbf{4 g}$ & $\mathrm{Cl}$ & 284 & 308 & 23692 & 24 \\
8 & $\mathbf{4 h}$ & $\mathrm{NHCO}_{2} \mathrm{Et}$ & 300 & 415 & 12550 & 115 \\
\hline
\end{tabular}

Solvent: chloroform + methanol $(1: 1, \mathrm{v} / \mathrm{v})$; concentration of 4: $10 \mathrm{ppm}$; Temperature $28-30{ }^{\circ} \mathrm{C}$.

\section{Conclusions}

We have developed a protocol for the synthesis of dibenzopyranones by the tandem Diels-Alder dehydrogenation reaction. DDQ in $o$-dichlorobenzene is the best dehydrogenating agent. These dibenzopyranones are pale yellow to yellow in color and show emission under UV light (365 $\mathrm{nm})$.

\section{Experimental Section}

General. The melting points were determined on an Analab melting point apparatus (Model$\mu$ Thermocal 10) in open capillary tubes and are uncorrected. The IR spectra were recorded on a Perkin-Elmer spectrum-100 FTIR spectrophotometer. The ${ }^{1} \mathrm{H}$ NMR spectra were recorded on Varian Mercury plus 300 (300 MHz) spectrometer in $\mathrm{CDCl}_{3} / \mathrm{DMSO}-\mathrm{d}_{6}$ with TMS as an internal standard and the chemical shifts are expressed in $\delta$ unit (ppm). The Mass spectra were recorded on a Finnigan LCQ Advantage Max spectrometer. Elemental analysis was carried out on a Thermo finnigan, Flash EA 1112. UV spectra were recorded on a Shimadzu UV-1601, UV-VIS Spectrophotometer. Fluorescence spectra were recorded on a Cary Eclipse fluorescence Spectrophotometer. Absorption and Emission spectra were performed using quartz cell (1 x 1 $\mathrm{cm})$. All compounds are excited at their absorption values shown in table no 6. Emission range was kept as 250-675 nm. Absorption and Emission spectra were performed using quartz cell ( $1 \mathrm{x}$ $1 \mathrm{~cm})$. Emission and excitation slit had the width equal to 5 constant for all measurements. 
Diels-Alder reaction followed by dehydrogenation reaction of 1a with NPMA (2) without DDQ in nitrobenzene. 1a $(0.262 \mathrm{~g}, 1 \mathrm{mmol})$ and NPMA $(0.519 \mathrm{~g}, 3 \mathrm{mmol})$ were refluxed in nitrobenzene $(5 \mathrm{~mL})$ for $24 \mathrm{~h}$. The solution was cooled to room temperature, $\mathrm{n}$-hexane $(15 \mathrm{~mL})$ was added and the solution stirred for $30 \mathrm{~min}$. The precipitated solid was filtered and subjected to column chromatography to obtain $\mathbf{4 a}$ using silica gel in chloroform.

\section{General procedure for dehydrogenation of Diels-Alder adduct (3) in nitrobenzene}

3 (1 mmol) was refluxed in nitrobenzene $(5 \mathrm{~mL})$ for $24 \mathrm{~h}$. The solution was cooled to room temperature. n-Hexane $(15 \mathrm{~mL})$ was added and the solution was stirred for $30 \mathrm{~min}$. The precipitated solid was filtered and subjected to column chromatography using silica gel in chloroform.

Tandem Diels-Alder dehydrogenation reaction of 1a in nitrobenzene. 1a $(0.262 \mathrm{~g}, 1 \mathrm{mmol})$, NPMA (0.519 g, $3 \mathrm{mmol})$ and DDQ (0.454 g, $2 \mathrm{mmol})$ were refluxed in nitrobenzene for $15 \mathrm{~min}$. After the completion of reaction, the solution was cooled to room temperature. n-Hexane (15 $\mathrm{mL}$ ) was added to the solution and stirred for $30 \mathrm{~min}$. The solvent was decanted and the insoluble mass was subjected to column chromatography using silica gel in chloroform.

General procedure for tandem Diels-Alder dehydrogenation reaction in $o$-dichlorobenzene 1 ( $1 \mathrm{mmol})$, NPMA $(0.519 \mathrm{~g}, 3 \mathrm{mmol})$ and DDQ $(0.454 \mathrm{~g}, 2 \mathrm{mmol})$ were refluxed in $o$ dichlorobenzene for $1 \mathrm{~h}$. After the completion of reaction, the solution was cooled to room temperature. Reduced DDQ was separated from the reaction mixture by filtration. n-Hexane (15 $\mathrm{mL}$ ) was added to the filtrate and stirred for $30 \mathrm{~min}$. The solvent was decanted and the insoluble mass was subjected to column chromatography using silica gel in chloroform.

$\boldsymbol{N}$-Phenyl-3-methyl-9-phenyl-6-oxo-6H-dibenzo $[\boldsymbol{b}, \boldsymbol{d}]$ pyran-7,8-dicarboximide (4a). Pale yellow solid; yield: 73\%; mp 272-273 ${ }^{\circ} \mathrm{C}$ (from $\mathrm{MeOH}$ ); IR (KBr): 1780, 1754, 1717 (CO), 1606, 1499 (aromatic C=C), $1384 \mathrm{~cm}^{-1} ;{ }^{1} \mathrm{H} \mathrm{NMR}\left(300 \mathrm{MHz}, \mathrm{CDCl}_{3}\right.$ ): $\delta 2.27$ (s, 3H, $\mathrm{C}_{3}$ ), 7.29$7.55(\mathrm{~m}, 12 \mathrm{H}, \mathrm{Ar} \underline{\mathrm{H}}), 8.02\left(\mathrm{~d}, J 8.7 \mathrm{~Hz}, 1 \mathrm{H}, \mathrm{C}_{1} \mathrm{H}\right), 8.35\left(\mathrm{~s}, 1 \mathrm{H}, \mathrm{C}_{10} \mathrm{H}\right)$; MS: $m / z 432.4(\mathrm{M}+\mathrm{H})$; Anal. Calcd. for $\mathrm{C}_{28} \mathrm{H}_{17} \mathrm{NO}_{4}$ : C, 77.95; H, 3.97; N, 3.25. Found: C, 77.87; H, 3.73; N, 3.12.

$\boldsymbol{N}$-Phenyl-3-hydroxy-9-phenyl-6-oxo-6H-dibenzo $[\boldsymbol{b}, \boldsymbol{d}]$ pyran-7,8-dicarboximide (4b). Yellow solid; yield: 71\%; mp 288-289 ${ }^{\circ} \mathrm{C}$ (from $\mathrm{MeOH}$ ); IR (KBr): $3120(\mathrm{OH}), 1775,1742,1720(\mathrm{CO})$, 1611 (aromatic C=C), $1374 \mathrm{~cm}^{-1} ;{ }^{1} \mathrm{H}$ NMR (400 MHz, DMSO-d 6 ): $\delta 6.76\left(\mathrm{~d}, J 2 \mathrm{~Hz}, 1 \mathrm{H}, \mathrm{C}_{4} \underline{\mathrm{H}}\right.$ ), $6.83\left(\mathrm{dd}, J 2\right.$ and $\left.8 \mathrm{~Hz}, 1 \mathrm{H}, \mathrm{C}_{2} \underline{\mathrm{H}}\right), 7.42-7.52(\mathrm{~m}, 8 \mathrm{H}, \mathrm{Ar} \underline{\mathrm{H}}), 7.72-7.73(\mathrm{bs}, 2 \mathrm{H}, \mathrm{Ar} \underline{\mathrm{H}}), 8.40$ (d, $J 8$ $\left.\mathrm{Hz}, 1 \mathrm{H}, \mathrm{C}_{1} \underline{\mathrm{H}}\right), 8.53\left(\mathrm{~s}, 1 \mathrm{H}, \mathrm{C}_{10} \underline{\mathrm{H}}\right), 10.68(\mathrm{~s}, 1 \mathrm{H}, \mathrm{OH}) ; \mathrm{MS}: \mathrm{m} / z 434.3(\mathrm{M}+\mathrm{H}) ;$ Anal. Calcd. for $\mathrm{C}_{27} \mathrm{H}_{15} \mathrm{NO}_{5}$ : C, 74.82; H, 3.49; N, 3.23. Found: C, 74.98; H, 3.35; N, 3.16.

$\boldsymbol{N}$-Phenyl-3-methoxy-9-phenyl-6-oxo-6H-dibenzo $[\boldsymbol{b}, \boldsymbol{d}]$ pyran-7,8-dicarboximide (4c). Pale yellow solid; yield: 69\%; mp 265-266 ${ }^{\circ} \mathrm{C}$ (from MeOH); IR (KBr): 1777, 1754, 1723 (CO), 1610

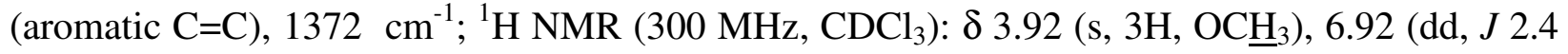
\& $\left.8.7 \mathrm{~Hz}, 1 \mathrm{H}, \mathrm{C}_{2} \underline{\mathrm{H}}\right), 6.96\left(\mathrm{~d}, J 2.4 \mathrm{~Hz}, 1 \mathrm{H}, \mathrm{C}_{4} \underline{\mathrm{H}}\right), 7.32-7.63(\mathrm{~m}, 10 \mathrm{H}, \mathrm{Ar} \underline{\mathrm{H}}), 8.00(\mathrm{~d}, J$ 8.7, 1H, 1$\mathrm{C} \underline{\mathrm{H}}), 8.27$ (s, $\left.1 \mathrm{H}, \mathrm{C}_{10} \underline{\mathrm{H}}\right)$; MS: $\mathrm{m} / z, 448.5(\mathrm{M}+\mathrm{H})$; Anal. Calcd. for $\mathrm{C}_{28} \mathrm{H}_{17} \mathrm{NO}_{5}$ : C, 75.16; H, 3.83; N, 3.13. Found: C, 75.08; H, 3.89; N, 3.16. 
$\boldsymbol{N}$-Phenyl-9-phenyl-6-oxo-6H-dibenzo[b,d]pyran-7,8-dicarboximide (4d). Pale yellow solid; yield: 66\%; mp 289-290 ${ }^{\circ} \mathrm{C}$ (from $\mathrm{CHCl}_{3}$ ); IR (KBr): 1782, 1761, 1720 (CO), 1608, 1503 (aromatic C=C), $1373 \mathrm{~cm}^{-1} ;{ }^{1} \mathrm{H}$ NMR $\left(300 \mathrm{MHz}, \mathrm{CDCl}_{3}\right): \delta 7.36-7.65(\mathrm{~m}, 13 \mathrm{H}, \mathrm{Ar} \underline{\mathrm{H}}), 8.13(\mathrm{~d}, J$ $\left.8.21 \mathrm{~Hz}, 1 \mathrm{H}, \mathrm{C}_{1} \underline{\mathrm{H}}\right), 8.42$ (s, $\left.1 \mathrm{H}, \mathrm{C}_{10} \underline{\mathrm{H}}\right)$; MS: $m / z 418.3(\mathrm{M}+\mathrm{H})$; Anal. Calcd. for $\mathrm{C}_{27} \mathrm{H}_{15} \mathrm{NO}_{4}$ : C, 77.69; H, 3.62; N, 3.36. Found: C, 77.50; H, 3.58; N, 3.21.

$\boldsymbol{N}$-Phenyl-9-phenyl-6-oxo-6H-dibenzo $[\boldsymbol{b}, \boldsymbol{d}]$ pyran-7,8-dicarboximide-3-yl tosylate (4e). Pale yellow solid; yield: 61\%; mp 275-276 ${ }^{\circ} \mathrm{C}$ (from $\mathrm{MeOH}$ ); IR (KBr): 1772, 1763, 1720 (CO), 1614, 1500 (aromatic C=C), $1376 \mathrm{~cm}^{-1} ;{ }^{1} \mathrm{H}$ NMR (300 MHz, $\left.\mathrm{CDCl}_{3}\right): \delta 2.48\left(\mathrm{~s}, 3 \mathrm{H}, \mathrm{CH}_{3}\right), 6.95$ $\left(\mathrm{d}, J 2.1 \mathrm{~Hz}, 1 \mathrm{H}, \mathrm{C}_{4} \underline{\mathrm{H}}\right), 7.19\left(\mathrm{dd}, J 2.1\right.$ and $\left.8.7 \mathrm{~Hz}, 1 \mathrm{H}, \mathrm{C}_{2} \underline{\mathrm{H}}\right), 7.35-7.62(\mathrm{~m}, 12 \mathrm{H}, \operatorname{Ar} \underline{\mathrm{H}}), 7.76(\mathrm{~d}$, $2 \mathrm{H}, \operatorname{Ar} \underline{\mathrm{H}}), 8.09$ (d, J $\left.8.7 \mathrm{~Hz}, 1 \mathrm{H}, \mathrm{C}_{1} \underline{\mathrm{H}}\right), 8.33$ (s, 1H, $\left.\mathrm{C}_{10} \underline{\mathrm{H}}\right)$; MS: m/z $588.6(\mathrm{M}+\mathrm{H})$; Anal. Calcd. for $\mathrm{C}_{34} \mathrm{H}_{21} \mathrm{NO}_{7} \mathrm{~S}$ : C, 69.50; H, 3.60; N, 2.38. Found: C, 69.38; H, 3.51; N, 2.48.

$\boldsymbol{N}$-Phenyl-9-phenyl-6-oxo-6H-dibenzo[ $[\boldsymbol{b}, \boldsymbol{d}]$ pyran-7,8-dicarboximide-7-yl acetate (4f). Yellow solid; yield: 63\%; mp 284-285 ${ }^{\circ} \mathrm{C}$ (from $\mathrm{MeOH}$ ); IR (KBr): 1780, 1761, 1721 (CO), 1609 (aromatic C=C), $1379 \mathrm{~cm}^{-1} ;{ }^{1} \mathrm{H}$ NMR $\left(300 \mathrm{MHz}, \mathrm{CDCl}_{3}\right.$ ): $\delta 2.36\left(\mathrm{~s}, 3 \mathrm{H}, \mathrm{CO}-\underline{\mathrm{H}}_{3}\right.$ ), 7.17 (dd, $J$ 2.4 and $\left.8.7 \mathrm{~Hz}, 1 \mathrm{H}, \mathrm{C}_{2} \underline{\mathrm{H}}\right), 7.25\left(\mathrm{~d}, J 2.4 \mathrm{~Hz}, 1 \mathrm{H}, \mathrm{C}_{4} \underline{\mathrm{H}}\right), 7.36-7.64(\mathrm{~m}, 10 \mathrm{H}, \mathrm{Ar} \underline{\mathrm{H}}), 8.12$ (d, J 8.7 $\left.\mathrm{Hz}, 1 \mathrm{H}, \mathrm{C}_{1} \underline{\mathrm{H}}\right) ; 8.35$ (s, $\left.1 \mathrm{H}, \mathrm{C}_{10} \underline{\mathrm{H}}\right) ; \mathrm{MS}: \mathrm{m} / z 476.6(\mathrm{M}+\mathrm{H})$; Anal. Calcd. for $\mathrm{C}_{29} \mathrm{H}_{17} \mathrm{NO}_{6}$ : C, 73.26; H, 3.60; N, 2.95. Found: C, 73.41; H, 3.44; N, 2.82.

$\boldsymbol{N}$-Phenyl-3-chloro-9-phenyl-6-oxo-6H-dibenzo $[\boldsymbol{b}, \boldsymbol{d}]$ pyran-7,8-dicarboximide $\quad(\mathbf{4 g}) . \quad$ Pale yellow solid; yield: 67\%; mp 296-297 ${ }^{\circ} \mathrm{C}$ (from MeOH); IR (KBr): 1777, 1760, 1724 (CO), 1607, 1500 (aromatic C=C), $1373 \mathrm{~cm}^{-1} ;{ }^{1} \mathrm{H}$ NMR (300 MHz, DMSO-d $\left.)_{6}\right): \delta 7.42-7.52(\mathrm{~m}, 9 \mathrm{H}$,

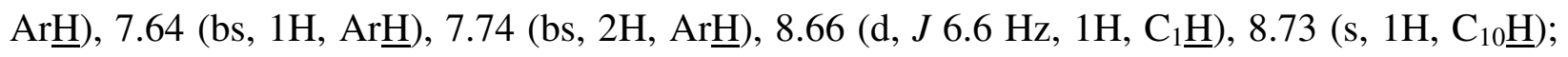
MS: $m / z 452.8(\mathrm{M}+\mathrm{H})$. Anal. Calcd. for $\mathrm{C}_{27} \mathrm{H}_{14} \mathrm{ClNO}_{4}$ : C, 71.77; H, 3.12; N, 3.10. Found: C, $71.63 ; \mathrm{H}, 3.19 ; \mathrm{N}, 3.12$.

$\boldsymbol{N}$-Phenyl-9-phenyl-6-oxo-6H-dibenzo $[\boldsymbol{b}, \boldsymbol{d}]$ pyran-7,8-dicarboximide-7-yl-ethylcarbamate

(4h). Yellow solid; yield: 72\%; mp > $300{ }^{\circ} \mathrm{C}$ (from $\mathrm{MeOH}$ ); IR (KBr): $3512(\mathrm{NH}), 1777,1755$, 1731 (CO), 1614, 1548 (aromatic C=C), 1367, $1234 \mathrm{~cm}^{-1} ;{ }^{1} \mathrm{H}$ NMR $\left(300 \mathrm{MHz}, \mathrm{CDCl}_{3}\right): \delta 1.28$

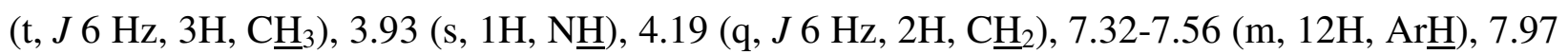
$\left(\mathrm{d}, J 8 \mathrm{~Hz}, 1 \mathrm{H}, \mathrm{C}_{1} \underline{\mathrm{H}}\right), 8.27\left(\mathrm{~s}, 1 \mathrm{H}, \mathrm{C}_{10} \underline{\mathrm{H}}\right)$; MS: $m / z 505.3(\mathrm{M}+\mathrm{H})$. Anal. Calcd. for $\mathrm{C}_{30} \mathrm{H}_{20} \mathrm{~N}_{2} \mathrm{O}_{6}: \mathrm{C}$, 71.42; H, 4.00; N, 5.55. Found: C, 71.32; H, 3.88; N, 5.42.

\section{Acknowledgements}

K.K.S. is thankful to the CSIR, New Delhi, for a fellowship.

\section{References}

1. Koch, K.; Podlech, J.; Pfeiffer, E.; Metzler, M. J. Org. Chem. 2005, 70, 3275. http://dx.doi.org/10.1021/j0050075r; PMid:15822993. 
2. Abe, H.; Nishioka, K.; Takeda, S.; Arai, M.; Takeuchi, Y.; Harayama, Tetrahedron Lett. 2005, 46, 3197. http://dx.doi.org/10.1016/j.tetlet.2005.03.046.

3. Sidwell, W. T. L.; Fritz H.; Tamm, C. Helv. Chim. Acta 1971, 54, 207. http://dx.doi.org/10.1002/hlca.19710540118.

4. Raistrick, H.; Stilkings, C. E.; Thomas, R. Biochemistry 1953, 55, 421.

5. Pero, R. W.; Harvan, D.; Blois, M. C. Tetrahedron Lett. 1973, 12, 945. http://dx.doi.org/10.1016/S0040-4039(00)72458-0.

6. Garino, C.; Bihel, F.; Pietrancosta, N.; Laras, Y.; Quelever, G.; Woo, I.; Klein, P.; Bain, J.; Boucher, J-L.; Kraus, J-L. Bioorg. Med. Chem. Lett. 2005, 15, 135. http://dx.doi.org/10.1016/j.bmcl.2004.10.018; PMid:15582426.

7. Sun, W.; Cama, L. D.; Birzin, E. T.; Warrier, S.; Locco, L.; Mosley, R.; Hammond, M. L.; Rohrer, S. P. Bioorg. Med. Chem. Lett. 2006, 16, 1468. http://dx.doi.org/10.1016/j.bmcl.2005.12.057; PMid:16412638.

8. Edwards, J. P.; West, S. J.; Marschke, K. B.; Mais, D. E.; Gottardis, M. M.; Jones, T. K. J. Med. Chem. 1998, 41, 303. http://dx.doi.org/10.1021/jm9705770; PMid:9464361.

9. Hamann, L. G.; Higuchi R. I.; Zhi, L.; Edwards, J. P.; Wang X-N.; Marschke, K. B.; Kong, J. W.; Farmer, L. J.; Jones, T. K. J. Med. Chem. 1998, 41, 623. http://dx.doi.org/10.1021/jm970699s; PMid:9484511.

10. Coghlan, M. J.; Kym, P. R.; Elmore, S. W.; Wang, A. X.; Luly, J. R.; Wilcox, D.; Stashko, M.; Lin, C.-W.; Miner, J.; Tyree, C.; Nakane, M.; Jacobson, P.; Lane, B. C. J. Med. Chem. 2001, 44, 2879. http://dx.doi.org/10.1021/jm010228c.

11. Schmidt, J. M.; Tremblay, G. B.; Page, M.; Mercure, J.; Feher, M.; Dunn-Dufault, R.; Peter, M. G.; Redden, P. R. J. Med. Chem. 2003, 46, 1289. http://dx.doi.org/10.1021/jm034007d; PMid:12672229.

12. Myrray, R.; Mendez, J.; Brown, S. The Natural Coumarins: Occurrence, Chemistry and Biochemistry; John Wiley \& Sons: New York, 1982; p 97.

13. Zhou, Q. J.; Worm, K.; Dolle, R. E. J. Org. Chem. 2004, 69, 5147. http://dx.doi.org/10.1002/ejic.200300320.

14. Kemperman, G. J.; Ter Horst, B.; Van de Goor, D.; Roeters, T.; Bergwerff, J.; Van der Eem R.; Basten, J. Eur. J. Org. Chem. 2006, 14, 3169. http://dx.doi.org/10.1002/ejoc.200600188.

15. Hussain, I.; Nguyen, V. T. H.; Yawer, T. T.; Fiscer, C.; Reinke, H., Langer, P. J. Org. Chem. 2007, 72, 6255. http://dx.doi.org/10.1021/jo070608r; PMid:17604399.

16. Alo, B. I.; Patil, P. A.; Sharp, M. J.; Siddiqui, M. A.; Snieckus, V. J. Org. Chem. 1991, 56, 3763. http://dx.doi.org/10.1021/jo00012a004.

17. Thasana, N.; Worayuthakarn, R.; Kradanrat, P.; Hohn, E.; Young, L.; Ruchirawat, S. J. Org. Chem. 2007, 72, 9379. http://dx.doi.org/10.1021/jo701599g; PMid:17975927.

18. Jung, M. E.; Allen, D. A. Org. Lett. 2009, 11, 757. http://dx.doi.org/10.1021/ol802792g; PMid:19138152. 
19. Bodwell, G. J.; Pi, Z.; Pottie, I. R. Synlett 1999, 477. http://dx.doi.org/10.1055/s-1999$\underline{2645}$.

20. Pottie, I. R.; Nandaluru, P. R.; Bodwell, G. J. Synlett 2011, 2245.

21. Pottie, I. R.; Nandaluru, P. R.; Benoit, W. L.; Miller, D. O.; Dawe, L. N.; Bodwell, G. J. J. Org. Chem. 2011, 76, 9015. http://dx.doi.org/10.1021/jo201775e; PMid:21955052.

22. Sanz, R.; Fernandez, Y.; Castroviejo, M. P.; Perez, A.; Fananas, F. J. Eur. J. Org. Chem. 2007, 1, 62. http://dx.doi.org/10.1002/ejoc.200600621.

23. Teske, J. A.; Deiters, A. Org. Lett. 2008, 10, 2195. http://dx.doi.org/10.1021/ol800589e; PMid:18452299.

24. Ku, Y-Y.; Grieme, T.; Raje, P.; Morton, H. E.; Rozema, M.; King, S. A. J. Org. Chem. 2003, 68, 3238. http://dx.doi.org/10.1021/jo0268613; PMid:12688796.

25. Mustafa, A.; Mohamad, K. J. Am. Chem. Soc. 1955, 77, 1828. http://dx.doi.org/10.1021/ja01612a034.

26. Mustafa, A.; Mohamad, K.; Mohmad, A. A. J. Am. Chem. Soc. 1956, 78, 4692. http://dx.doi.org/10.1021/ja01599a044.

27. Soliman, A. Y.; El-Kafrawy, A. F.; Mohamed, F. K.; Baker, H. M. Abdel-Gawad, A. M. Indian. J. Chem. 1991, 30B, 477.

28. Shridhar, D. R.; Reddy Sastry, C. V.; Vaidya, N. K.; Moorty, S. R.; Reddi, G. S.; Thapar, G. S. Indian. J. Chem. 1978, 16B, 704.

29. Allum, K. G.; Howell, I. V.; Pitkethly, R. C.; Brit. Pat.; 1,378,151, 1974; (Chem. Abstr. 1975, 82, 125043r).

30. Rozhdestvenskaya, N. N.; Isagulyanto, G. V.; Kupcha, L. A.; Kozlov, N. S.; Izv. Akad. Nauk SSSR, Ser. Khim. 1991, 1751 (Chem. Abs. 1992, 116, 20722d).

31. Haber, J.; Wojciechowska, M.; Gut, W. Bull. Pol. Acad. Sci. Chem. 1996, 44, 55 (Chem. Abs. 1996, 125, 301640a).

32. Kozlov, N. S.; Zhavnerlo, K.; Kozlyak, M. I. Dokl. Akad. Nauk Beloruss SSR 1970, 14, 914 (Chem. Abs. 1971, 74, 76099n).

33. Kim, H. S.; Kim, D. I.; Kim, C-S.; Joo, Y. J.; Kongop Hwahak 1994, 5, 871 (Chem. Abs. 1995, 123, 143142s).

34. Clarke, H. T.; Davis, A. W. Org. Synth. Coll. Vol. 1932, 1, 478. 